\title{
Temporal variations in the magnetic network large-scale dynamics
}

\author{
N. Meunier ${ }^{1}$ \\ Laboratoire d'Astrophysique de l'Observatoire Midi-Pyrénées, 57 avenue d'Azereix, BP 826, 65008 Tarbes Cedex, France \\ e-mail: meunier@bagn.obs-mip.fr
}

Received 15 April 2005 / Accepted 26 July 2005

\section{ABSTRACT}

The variation in the magnetic network large-scale dynamics is investigated using MDI magnetograms from 1996 to 2004 . Cross-correlation and feature tracking techniques are used on pairs of magnetograms in order to derive the dynamics at the solar surface, including magnetic network regions. The variations for both methods are very different, the feature tracking technique leading to generally much less variable dynamics over the cycle, except for the rotation versus latitude. At cycle minimum, the increased differential rotation at high latitude combined with a more rigid rotation at low latitude is indeed more pronounced with the feature tracking. The rotation variations put a strong constraint on the origin of these variations, as some strong correlations between coefficients deduced from fits with Legendre polynomials are observed. When using the cross-correlation technique, the meridional circulation also tends to exhibit a more complex behavior compared to rotation. This study provides the temporal variations in the dependence of the dynamics on the magnetic field and feature size as well as on polarities. The dispersions in velocity for the following and leading polarities are studied over the cycle.

Key words. Sun: magnetic fields - Sun: photosphere - Sun: activity - Sun: faculae, plages

\section{Introduction}

The large-scale dynamics of the solar surface plays an important role in the solar cycle processes. The angular rotation velocity (hereafter rotation rate), the meridional circulation and the supergranular diffusion at the solar surface have a strong impact on the solar dynamo and on the spatial distribution of the magnetic field on the surface of the Sun. As a consequence, the variation in this dynamic with time is important to constrain the dynamo models, and in particular the possible retroaction of the dynamo on the flows.

For that purpose, many works have been done in the past concerning the variation in sunspot rotation (e.g. Balthasar \& Wöhl 1980; Arévalo et al. 1982; Lustig 1983; Gilman \& Howard 1984; Balthasar et al. 1986; Vazquez \& Wöhl 1986; Hathaway \& Wilson 1990; Kambry \& Nishikawa 1990; Nesme-Ribes et al. 1993; 1997). The variations in sunspot meridional circulation have been more difficult to detect (Balthasar \& Wöhl 1980; Arévalo et al. 1982; Hanslmeier \& Lustig 1986; Howard \& Gilman 1986; Lustig \& Hanslmeier 1987; Javaraiah 1999). Some work has also been done to use local helioseismology to determine the variation in largescale flows just below the surface of the Sun (Chou \& Dai 2001; Haber et al. 2002; Basu \& Antia 2003; Zhao \& Kosovichev 2004). A complementary approach is the study of the magnetic network dynamics. Komm et al. (1993a,b) attempted this study but the uncertainties were still quite large.
Meunier et al. (1997a,b) also studied the variation in rotation and meridional circulation with time, using a manual feature tracking. Using a more extensive data set, Meunier (1999, 2005a) used cross-correlation and feature tracking between pairs of MDI full-disk magnetograms. Meunier (2005a) was able to use this large data set to study the variation in the dynamics with various physical parameters such as magnetic field or structure size. Meunier (2005b) used the same large data set to study the dependence on polarities.

In this work we investigate the variation in the magnetic network dynamics over almost a complete solar cycle using the same techniques on this large data set. This allows the stability of the new results obtained by Meunier (2005a) to be tested, such as the variation in dynamics with the local magnetic field, and by Meunier (2005b), such as the difference in dynamics between the leading and following polarity areas in weak field regions. This approach should also provide some clues to better understand these results. The data processing is briefly described in Sect. 2. The variations in angular velocity are described in Sect. 3 and those in meridional circulation in Sect. 4. The new results are discussed in Sect. 5.

\section{Data and processing}

The time series used in Meunier (2005a,b) is used here to study the temporal variation in the dynamics of the magnetic network. Pairs of full-disk MDI magnetograms covering 8 years 
are analyzed, from June 1996 to April 2004. Only 5-min magnetograms ${ }^{1}$ at the 1.8 level, i.e. the latest level provided by the $\mathrm{MDI} / \mathrm{SOHO}$ team, are considered in this study. Their temporal distribution is shown in Meunier (2005b). Pairs of magnetograms are separated by 96 minutes. The dynamics were derived using two complementary techniques. First, a crosscorrelation technique over large boxes $\left(7.68 \times 7.68 \mathrm{deg}^{2}\right)$, hereafter CT, was used. The output consists of maps of horizontal velocities in both directions (rotation and meridional circulation). The average unsigned magnetic field $\left\langle\left|B_{\mathrm{c}}\right|\right\rangle$ is computed over each box, as well as the unsigned maximum magnetic field $\left\langle\left|B_{\mathrm{mc}}\right|\right\rangle$ averaged over the box. Second, a feature tracking analysis, hereafter FT, is used. Magnetic structures are defined by adjacent pixels with magnetic field above a threshold of $40 \mathrm{G}$ (or below $-40 \mathrm{G}$ for negative magnetic fields). These structures are then tracked between pairs of magnetograms separated by 96 min as for CT. The output of FT is a list of structures associated with their properties (such as size or average magnetic field) and velocities (rotation and meridional circulation as for CT). We refer to Meunier (2005a) for more details, as the analysis is exactly the same. The dynamics is then computed for each year separately, or as a function of time with a small time step. In this paper, we are mostly interested in the network dynamics. As a consequence, only the CT boxes with $\left\langle\left|B_{\mathrm{c}}\right|\right\rangle$ lower than $20 \mathrm{G}$ are considered, as well as FT structures with a size lower than $100 \mathrm{Mm}^{2}$ for FT (Wang 1988; Meunier 2003). These 2 thresholds correspond well to each other (Meunier 2005a). Note that the results are dominated by the weaker field regions.

\section{Variation in the angular velocity}

\subsection{Variations in latitude}

Fits using Legendre polynomial in $\sin ^{i}(\theta)$ ( $\theta$ the latitude) up to the 4th degree are performed on the rotation rates versus latitude. The variations in these coefficients with time are shown in Fig. 1 for the CT and FT methods. The variation in the average magnetic field (for all CT boxes) is also shown for comparison and allows the identification of typical phases of the solar cycle, such as cycle minimum, the rising phase, cycle maximum and the declining phase. The values for cycle minimum and cycle maximum are also shown in Table 1 . The equatorial rotation rate, deduced from $A_{0}, A_{2}$ and $A_{4}$, is shown in Fig. 2 for CT. The FT rate is not shown because the large errorbars on $A_{2}$ and $A_{4}$ with that technique lead to large errorbars on the equatorial rate as well. However, the variation deduced from a fit directly using polynomials in $\sin ^{i}(\theta)$ is also shown. In the CT case, they give a variation similar to that deduced from the Legendre polynomials.

The CT equatorial rotation rate is larger at cycle maximum than at cycle minimum. However, the variation is far from monotonous from minimum to maximum, as it starts to decrease from cycle minimum to the middle of the rising phase (this was already seen by Meunier 1999), and then increases toward larger values at cycle maximum. The FT equatorial rate

\footnotetext{
1 The averaged magnetogram is derived from 5 single magnetograms covering $5 \mathrm{~min}$.
}
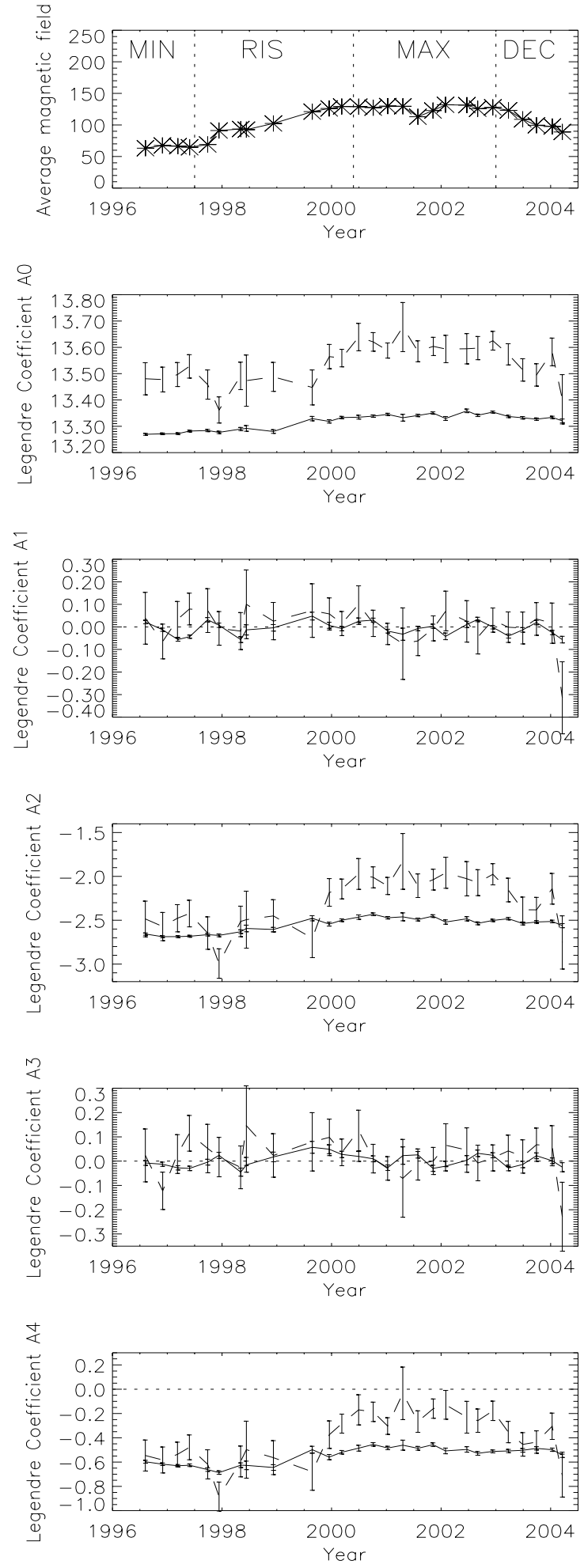

Fig. 1. Top: average CT unsigned magnetic field versus time. Following plots: temporal variation in the coefficients from Legendre polynomial fits on the rotation rates versus latitude (in deg/day) for CT (solid lines) and FT (dashed lines).

shows a similar variation at the beginning of the cycle, but the subsequent increase is smaller than for CT as shown in Fig. 2. This is confirmed by the equatorial rate values in Table 1 . 
t1

Table 1. Coefficients $A_{0}$ to $A_{4}$ from fits with Legendre polynomials in $\sin ^{i}(\theta)$ on rotation rates versus latitude (in deg/day) and equatorial rate, $A_{\text {eq }}$, from a polynomial fit in $\sin ^{i}(\theta)$, for CT and FT at cycle minimum and cycle maximum.

\begin{tabular}{ccccccc}
\hline \hline Mode & $A_{0}$ & $A_{1}$ & $A_{2}$ & $A_{3}$ & $A_{4}$ & $A_{\text {eq }}$ \\
\hline CT min & $13.274 \pm 0.002$ & $-0.027 \pm 0.004$ & $-2.678 \pm 0.006$ & $-0.019 \pm 0.005$ & $-0.617 \pm 0.006$ & $14.381 \pm 0.002$ \\
CT max & $13.339 \pm 0.001$ & $-0.0005 \pm 0.0026$ & $-2.493 \pm 0.004$ & $0.006 \pm 0.003$ & $-0.497 \pm 0.004$ & $14.400 \pm 0.001$ \\
FT min & $13.48 \pm 0.02$ & $0.02 \pm 0.03$ & $-2.53 \pm 0.07$ & $0.02 \pm 0.04$ & $-0.57 \pm 0.05$ & $14.535 \pm 0.006$ \\
FT max & $13.58 \pm 0.01$ & $0.01 \pm 0.01$ & $-2.13 \pm 0.03$ & $0.03 \pm 0.01$ & $-0.28 \pm 0.02$ & $14.535 \pm 0.003$ \\
\hline
\end{tabular}

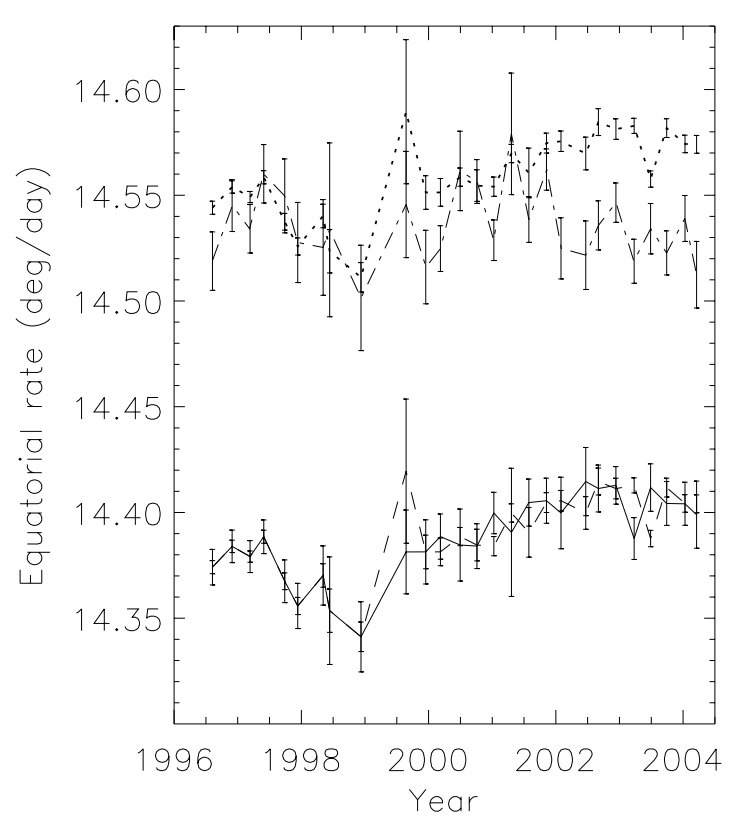

Fig. 2. Equatorial rotation rate versus time (in deg/day), for CT derived from the Legendre polynomial fit (solid line), for CT derived from the $\sin ^{i}(\theta)$ fit (dashed line), for FT derived from the $\sin ^{i}(\theta)$ fit (dotteddashed line). The dotted curve is the same as the dashed line shifted by $0.17 \mathrm{deg} /$ day so as to match approximately the FT curve around solar minimum.

The variation until 1999 is however very well correlated between the two techniques.

Furthermore, for both CT and FT, the absolute values of $A_{2}$ and $A_{4}$ are decreasing, which means that the rotation is also more rigid at cycle maximum compared to cycle minimum and to the rising phase. The correlations of $A_{2}$ and $A_{4}$ with the activity level are quite good. However, the amplitude of the variation is much larger for FT than for CT as shown in Fig. 1 and in Table 1. Figure 3 also shows the difference between the polynomials fits at cycle minimum and cycle maximum for both techniques. The more differential rotation at cycle minimum is mostly present at high latitude above $40^{\circ}$. At low latitude, the rotation is less differential at cycle minimum, although it is less significant.

An interesting feature in Fig. 1 is that $A_{2}$ and $A_{4}$ variations are strongly correlated. This is true for both CT (correlation coefficient 0.94 and 0.67 respectively) and FT (correlation coefficient 0.99 and 0.94 respectively). This is surprising because the Legendre polynomial are orthogonal to each other which means that there is no correlation between coefficients due to the fitting technique. The only reason for such a correlation is that various domains in latitude must have an actual correlated behavior. The effect of the negative $A_{4}$ term is to provide a more rigid rotation at low latitudes (in the range $\pm 20^{\circ}$ ) compared to what it would be with $A_{2}$ only, and a more differential rotation at high latitudes. So the more rigid rotation at cycle maximum at high latitude is physically correlated with a less rigid rotation at low latitude. This constrains a lot the processes at the origin of this variability. On the other hand, a plot of all polynomials for each period shows that the variations in rotation around latitudes $35-40^{\circ}$, i.e. the activity belt limit, in both hemispheres is very small, especially for FT.

On the other hand, Fig. 1 shows that the antisymmetry between hemispheres remains very small during the whole period. Coefficients $A_{1}$ and $A_{3}$ are also strongly correlated although very small in amplitude. However, when averaging over a longer period, it is possible to detect some antisymmetry. Table 1 shows that there is a significant antisymmetry with CT at cycle minimum, as the Southern hemisphere rotates faster (see also Fig. 3). This is not the case at cycle maximum. This antisymmetry could be related to the similar antisymmetry, although of larger amplitude, detected by Meunier (2005c) in coronal holes at high latitude. For FT, coefficients $A_{1}$ and $A_{3}$ are more uncertain and therefore it is more difficult to conclude. There may be an antisymmetry in the other direction (the Northern hemisphere rotating faster than the Southern hemisphere) with FT at cycle maximum, however.

\subsection{Size and magnetic field dependence}

In each latitude bin, the average rotation rate is computed and subtracted from the individual rotation rates, providing a residual rotation rate. Meunier (2005a) found that the residual rotation rates increased with increasing size of the magnetic features when using FT (up to $40 \mathrm{Mm}^{2}$ ) and with increasing magnetic field when using CT (for $\left\langle\left|B_{\mathrm{c}}\right|\right\rangle$ up to $\sim 8-9 \mathrm{G}$ ). The residuals then decreased strongly down to smaller rotation rates for active regions. Here the emphasis is put on weak field regions. Figure 4 shows the variation in rotation residuals for each year and for both methods. It shows that the variation in rotation with size observed by Meunier (2005a) for the FT method exhibits the same pattern at all time. However, the curves for the CT method are much more variable with time. The pattern averaged over the whole period observed by Meunier (2005a) is indeed observed in 1997 and 1998 for example and is in agreement with the previous result, but it is absent in 2003 and even reversed in 2004. 

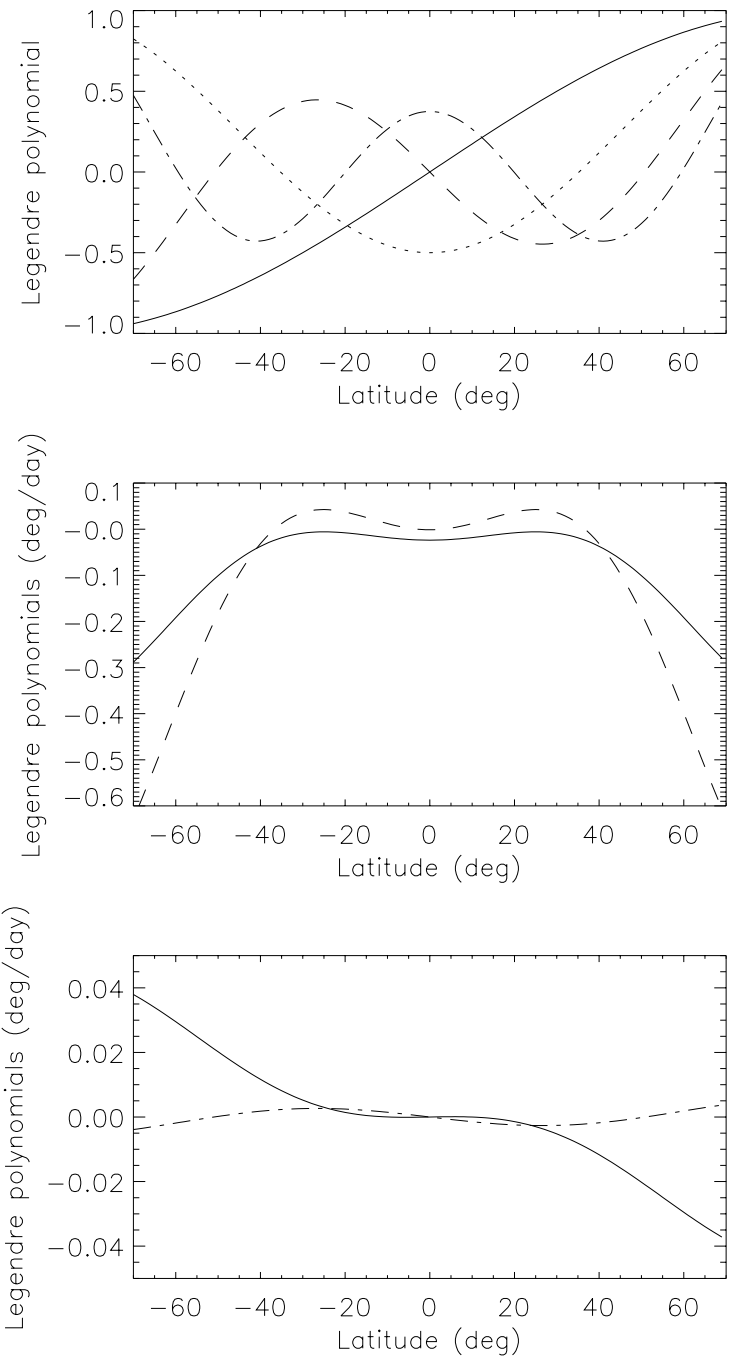

Fig. 3. Top: Legendre polynomial versus latitude, for degree 1 (solid line), degree 2 (dotted line), degree 3 (dashed line), and degree 4 (dotted-dashed line). Middle: symmetrical part of the Legendre polynomial fit difference $\Omega_{\min }-\Omega_{\max }$ between cycle minimum and cycle maximum for CT (solid line) and FT (dashed line), with $\Omega$ the symmetrical part of the Legendre polynomial fit of the rotation rate versus latitude. Bottom: antisymmetrical part of the Legendre polynomial fit for $\mathrm{CT}$ at cycle minimum (solid line) and cycle maximum (dotteddashed line).

\subsection{Polarities}

Meunier (2005b) found that weak-field regions of following polarity were rotating faster than regions of leading polarity when using the CT method. This was not observed with the FT method. Independently, a faster rotation at the location of following polarity magnetic fields has been observed by Zhao et al. (2004) when using a helioseismologic time-distance analysis to derive the large-scale flows, with a larger amplitude than in Meunier (2005b). They also observed a temporal variation in amplitude of this effect. It is therefore interesting to compare both temporal variations to see whether they correspond to the same phenomenon.

Figure 5 shows the amplitude of the difference between following and leading residual rotation rates for $\mathrm{CT}$, in both
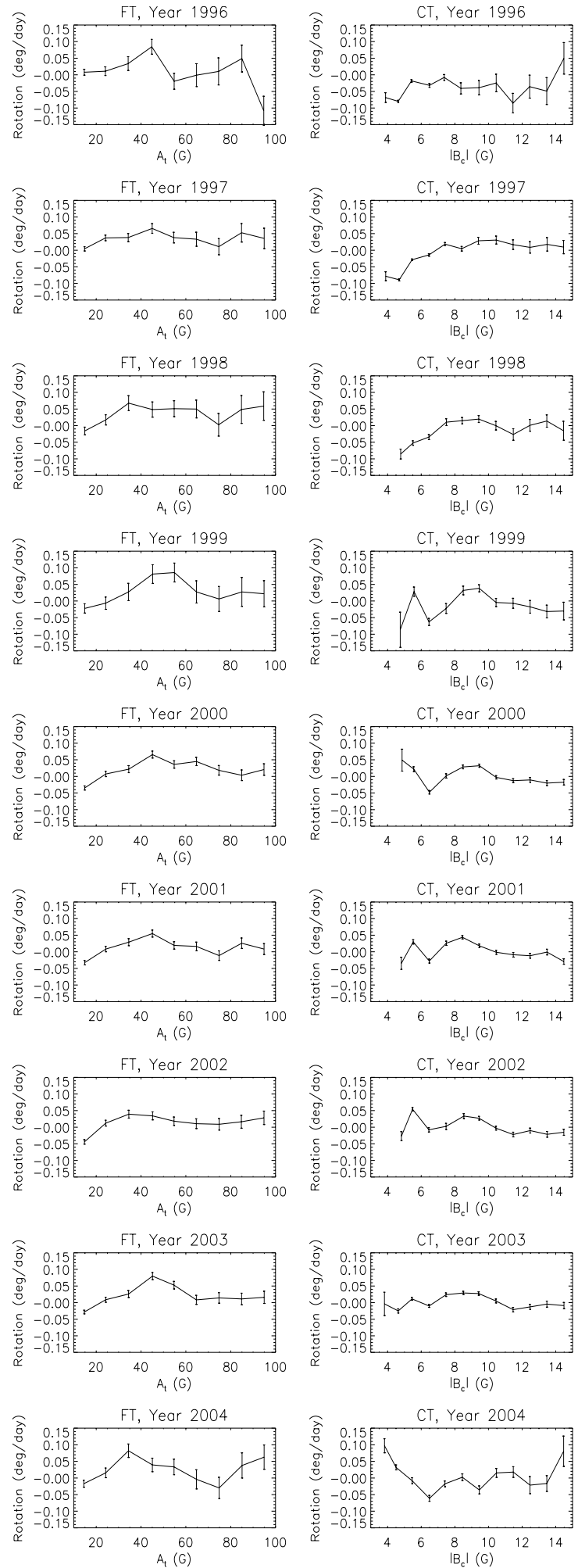

Fig. 4. Left: FT residual rotation rates versus feature size $A_{t}$ from 1996 (top) to 2004 (bottom). Right: same for CT residual rotation rates versus the average unsigned magnetic field $\left\langle\left|B_{\mathrm{c}}\right|\right\rangle$.

hemispheres. A first plot shows the average of residuals for $\left|\left\langle B_{\mathrm{c}}\right\rangle\right| /\left\langle\left|B_{\mathrm{c}}\right|\right\rangle$ in the range $0.2-0.7$, where the effect is the largest. This is similar to the computation made in Meunier (2005b) except that the variation is computed for each year separately and averaged over $\left|\left\langle B_{\mathrm{c}}\right\rangle\right| /\left\langle\left|B_{\mathrm{c}}\right|\right\rangle$. The second plot shows 

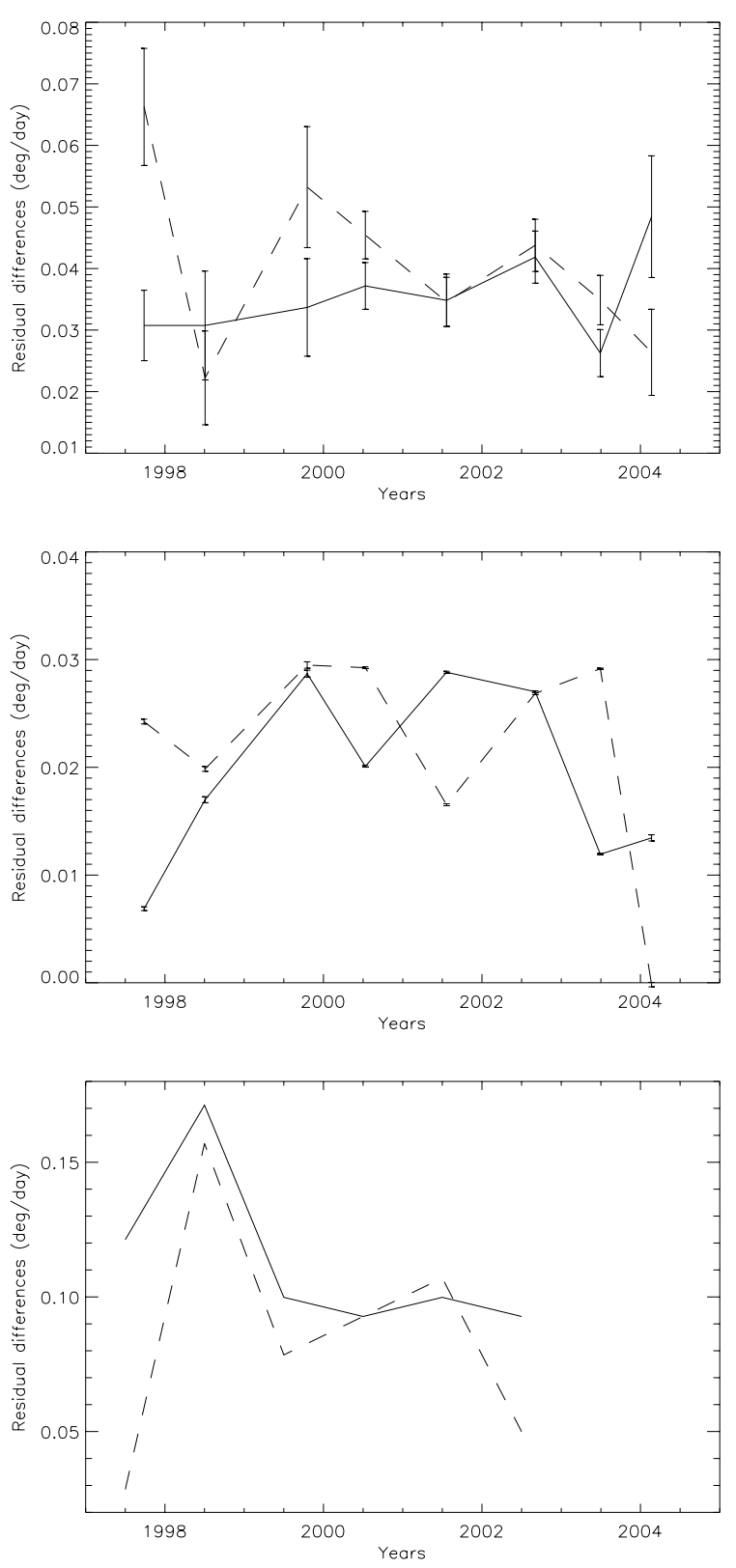

Fig. 5. Difference between the rotation residual for following and leading polarity in the Northern hemisphere (solid lines) and Southern hemisphere (dashed lines), versus time. Top: averages computed for $\left|\left\langle B_{\mathrm{c}}\right\rangle\right| /\left\langle\left|B_{\mathrm{c}}\right|\right\rangle$ in the range $0.2-0.7$. Middle: position of the Gaussian fit on the distribution of residuals, in the same conditions. Bottom: residual differences from Zhao et al. (2004) for a magnetic field of $100 \mathrm{G}$.

the location of a Gaussian fit on the distribution of the residual rotation rates, instead of the average. This is justified by the fact that these distributions exhibit large tails, as shown in Fig. 6, and it is interesting to see which part of the distribution contributes the most to the following-leading difference. On average, the points corresponding to the Gaussian distribution (90\% of the whole set) contribute to approximately $60 \%$ of the following-leading difference. Therefore the points in the tails of the distribution contribute more than expected from the percentage of points they represent $(10 \%)$. The errorbars in the first panels of Fig. 5 correspond to the statistical errors at the
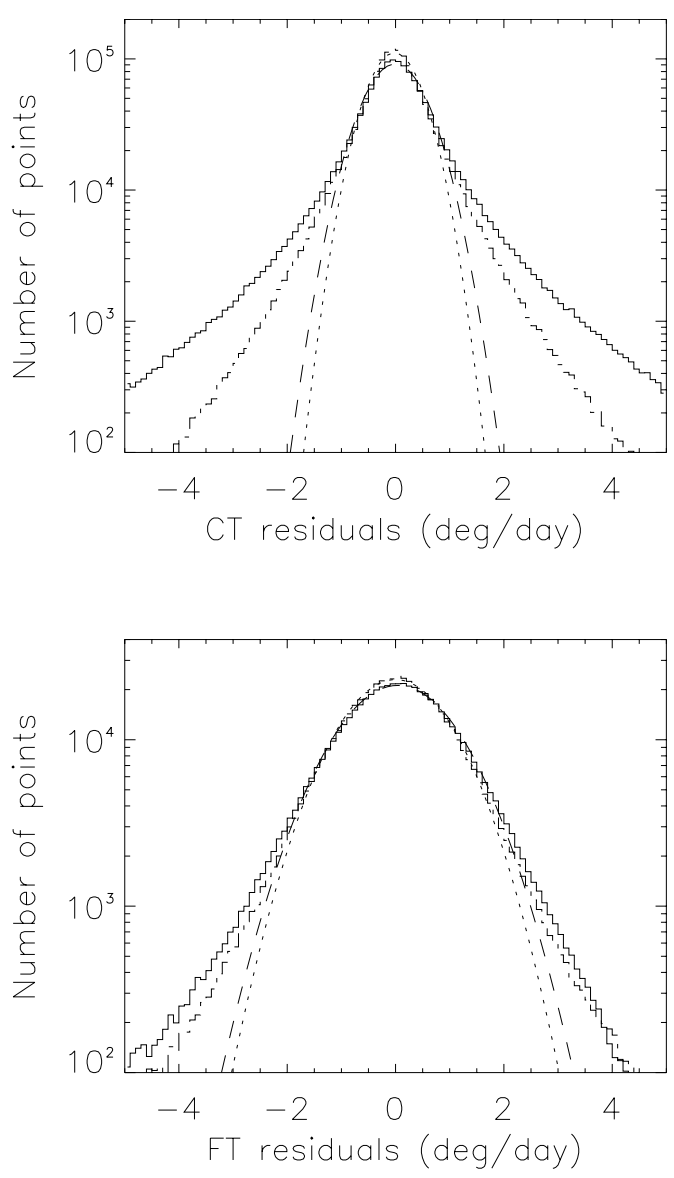

Fig. 6. Top: distribution of CT rotation residual (solid line) and CT meridional circulation (dotted-dashed line). Gaussian fits on these distribution are shown as dashed and dotted lines respectively. Bottom: same for FT.

$1-\sigma$ confidence level. In the second panel, they correspond to the uncertainty on the Gaussian fit at the $1-\sigma$ confidence level.

Figure 5 also shows a comparison with the Zhao et al. (2004) results. The values are deduced from their plots for a $100 \mathrm{G}$ magnetic field. For this value, the errorbars on the residual velocity for each polarity are of the order of $2.8 \mathrm{~m} / \mathrm{s}$, leading to a typical uncertainty on the differences plotted in Fig. 5 of the order of $0.03 \mathrm{deg} / \mathrm{day}$. At lower magnetic fields, the difference between following and leading residual rotation rates is not very different because the slope on the following polarity side is steep. The variations are very different for the various approaches, as none of the results obtained with the average or the Gaussian fit on the distribution have a good correlation with the results of Zhao et al. (2004). However, the errorbars on the Zhao et al. (2004) results are quite large; it is therefore difficult to compare the variations. Only between 1997 and 1998 does there seem to be a significantly different trend.

No significant difference between following and leading regions could be observed for small structures in Meunier (2005b) when using the FT technique. When looking at the temporal variation, the result remains the same and I do not identify any period with a significant signal. 

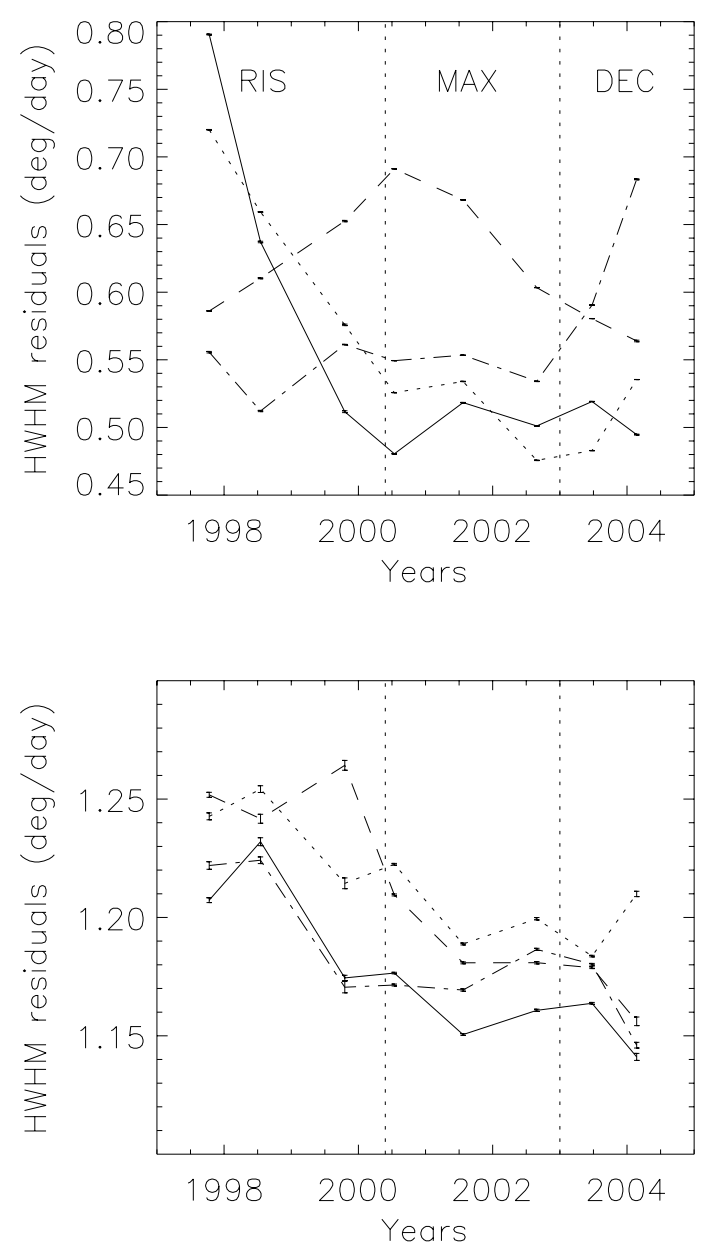

Fig. 7. Top: HWHM on CT rotation residual distributions versus time, for the leading polarity in the Northern hemisphere (solid line), following polarity in the Northern hemisphere (dashed line), leading polarity in the Southern hemisphere (dotted line) and following polarity in the Southern hemisphere (dotted-dashed line). Bottom: same for the FT method.

\subsection{Velocity dispersions}

The tails of the distribution as shown in Fig. 6 extend further for the rotation than the meridional circulation. The effect is more pronounced for CT. Such a difference between rotation and meridional circulation could be due to the differential rotation inside each bin. For this reason we expect a stronger effect for FT. As FT concerns individual features and CT results from a weighted average over a larger box which may include several features, we expect CT velocities to exhibit smaller dispersions. The results are not compatible with this. We also find that on average, the width of the Gaussian fit is larger for FT than for CT, despite the fact that the tails have a smaller extent. It is likely that the larger width in FT is related to the diffusion of magnetic features at the surface. The CT residuals are related to large-scale flows (such as those studied by Ambrǒz 2001), and the results suggest that $\mathrm{CT}$ provides stronger large-scale flows.

Figure 7 shows the HWHM of the residuals for both polarities in both hemispheres. For the CT method, leading polarity regions exhibit large HWHM at cycle minimum. The HWHM then decreases towards lower values by a factor of 2 during the maximum and declining phase. On the other hand, the following polarity starts with low values, which then increase during cycle maximum and the declining phase of the cycle. As a consequence the 2 categories of curves (leading and following) cross each other, just before the solar maximum. The Northern hemisphere is in advance by about 1 year compared to the Southern hemisphere. The rms values over the whole series of residuals show the same behavior although shifted in time by about 6 months to 1 year. The percentage of points in the tails compared to the core is well correlated with these curves. Therefore, large-scale flows exhibit large variations over the solar cycle, a large North-South asymmetry and large differences between polarities. At the end of the cycle the following polarity will become the leading polarity and vice-versa. So a cyclic behavior must be observed and this will have to be checked with a longer data set.

For the FT method, there is a large North-South asymmetry as well. However, there is no clear difference between leading and following polarities. From the beginning of the rising phase to the declining phase, a decrease in HWHM is observed.

\section{Variation in the meridional circulation}

\subsection{Variations in latitude}

Figure 8 shows the meridional circulation versus latitude for two years, in 1997 (at the end of solar minimum) and in 2004 (just after solar maximum). The FT meridional circulation is not very different in the two plots. On the other hand, the CT meridional circulation, larger in amplitude, is very different, especially at high latitudes. Another way to visualize these variations is to look at the temporal variation separately for each latitude bin, as shown in Fig. 9. At low latitudes (roughly in the activity belt), there is a correlation showing that the CT meridional circulation is evolving similarly in both hemispheres. Most of the signal is due to the propagation of the strong converging flows observed by Meunier $(1999,2005$ a) toward the equator. The FT meridional circulation remains very small, except around $15^{\circ}$, with a more poleward motion at cycle minimum.

At high latitude, i.e. above $60^{\circ}$, it is completely different. In the Northern hemisphere, the large poleward circulation decreases toward much smaller values at the end of cycle maximum. On the other hand, in the Southern hemisphere, the small poleward circulation increases during the whole period.

\subsection{Polarities}

Meunier (2005b) has detected some differences in meridional circulation between following and leading polarity weak field regions. For the weakest fields there was a strong asymmetry between hemispheres, with a more poleward motion of following polarity in the Southern hemisphere, while the opposite was observed in the Northern hemisphere. Some variations with latitudes were also observed. Figure 10 shows the variation in the following-leading difference with time, for $\left|\left\langle B_{\mathrm{c}}\right\rangle\right| /\left\langle\left|B_{\mathrm{c}}\right|\right\rangle$ in the range $0.2-0.7$. The variations in average and Gaussian fit position are well correlated in the Northern hemisphere 

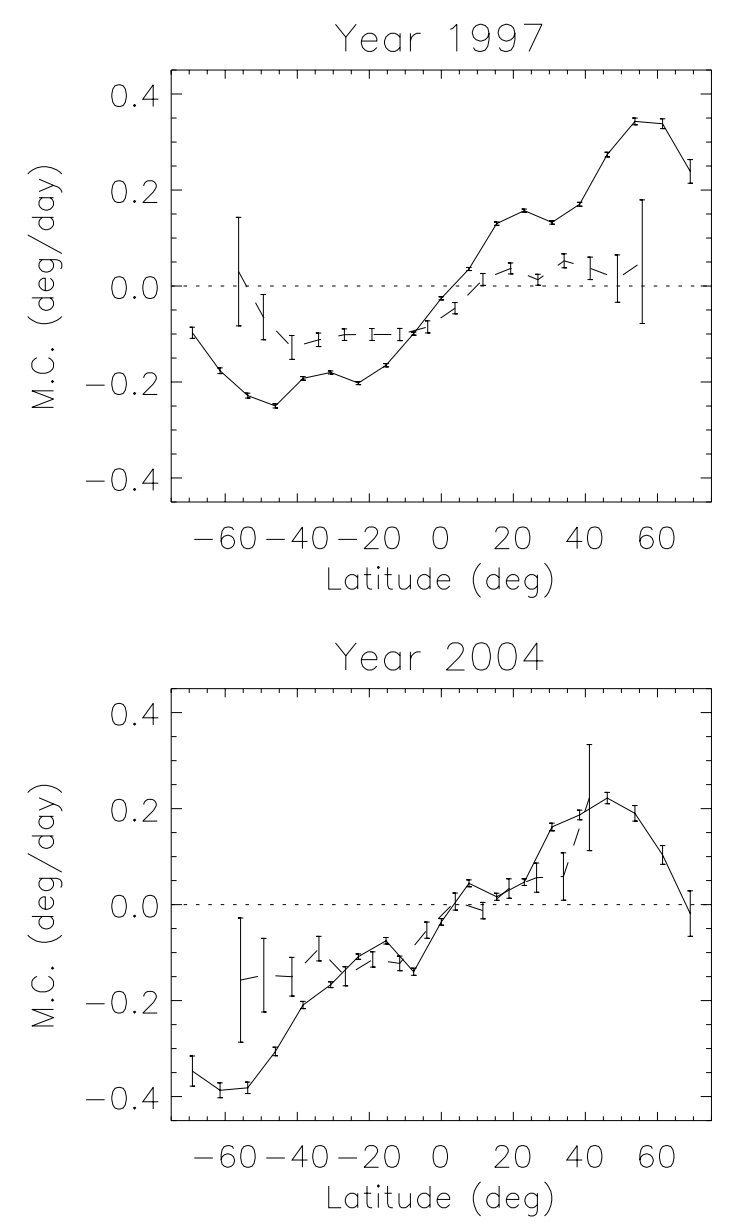

Fig. 8. CT (solid line) and FT (dashed line) meridional circulations versus latitude, for 1997 and 2004. Positive meridional circulation is towards the North pole.

(correlation of 0.84 ) but they are not as well correlated in the Southern hemisphere (correlation of 0.44). The Gaussian fit tends to provide a smaller difference than the average, as for rotation. On the other hand, there is a strong NorthSouth asymmetry, as the following polarity tends to have a more poleward motion in the Southern and a less poleward motion in the Northern hemisphere, as detected by Meunier (2005b). However, this is not true at all time, as there are periods with a more poleward motion for the following polarity in the Northern hemisphere, and a tendency for a less poleward motion in the Southern hemisphere as deduced from the Gaussian fit in 1998 and 2003. The correlation between the 2 hemispheres is significant for the average (correlation of 0.56 ) but smaller for the fit position (correlation of 0.28).

\subsection{Velocity dispersions}

As for rotation, Fig. 11 shows the HWHM on the residual distribution for the CT techniques and FT techniques. The results are qualitatively similar to those obtained for rotation in the previous section.
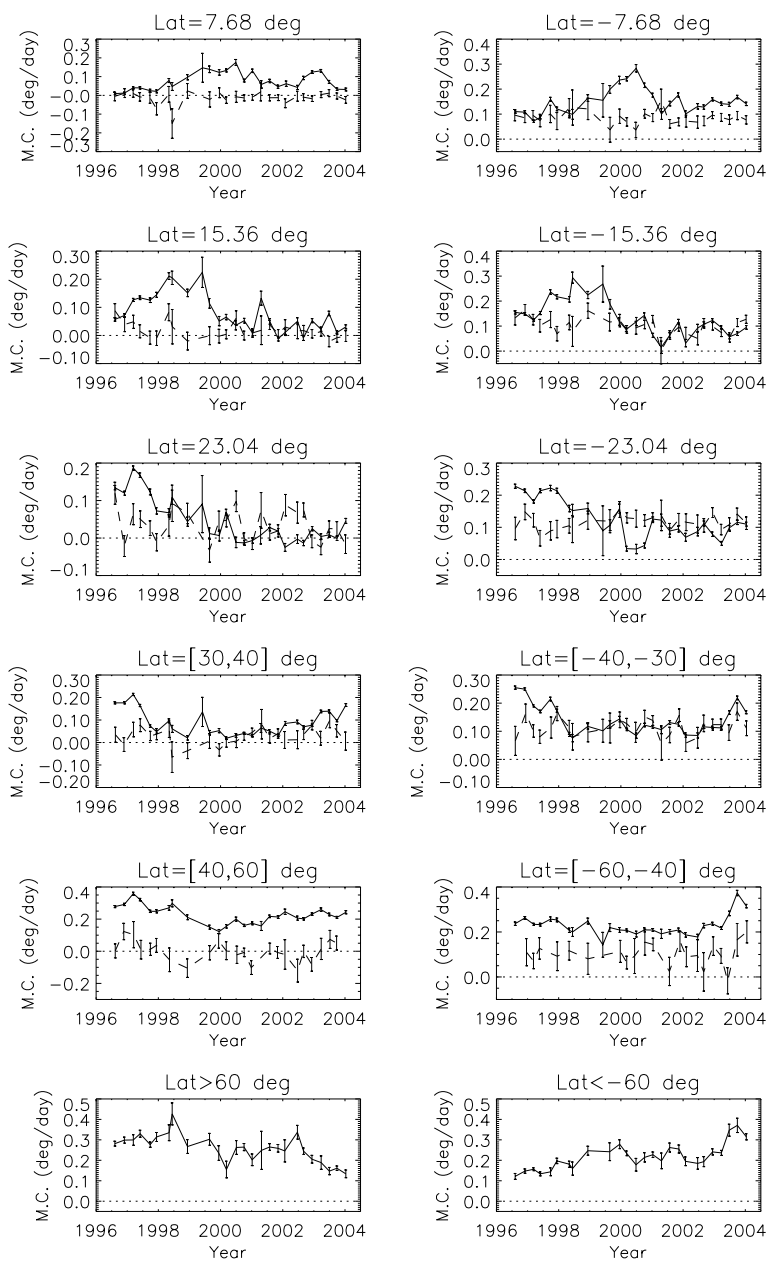

Fig. 9. CT (solid line) and FT (dashed line) meridional circulations versus time for various latitude ranges, represented on the same scale. The latitude values in the latitude belt correspond to the central values of the bin used to compute the CT boxes. Left: northern hemisphere. Right: southern hemisphere. Positive meridional circulation is towards the poles.

\section{Discussion and conclusion}

It is interesting to compare the magnetic network dynamics with sunspots and plage dynamics. The variations in sunspot rotation have been studied by many authors, and there is a good agreement for a more rigid rotation during cycle maximum (Balthasar \& Wöhl 1980; Lustig 1983; Nesme-Ribes et al. 1993), especially at the end of the cycle (Balthasar \& Wöhl 1980). This is consistent with the result obtained here for the magnetic network. Many works on sunspots rotation have found more equatorial rotation at cycle minimum (Balthasar \& Wöhl 1980; Lustig 1983; Gilman \& Howard 1984; Balthasar et al. 1986; Vazquez \& Wöhl 1986; Hathaway \& Wilson 1990; Kambry \& Nishikawa 1990). Furthermore, most of them have found a second peak in the equatorial rotation during cycle maximum, the lowest rotation rate occuring between cycle minimum and cycle maximum. In that case again, there is a qualitative agreement with the results for the magnetic network obtained here. However, the high equatorial rotation during cycle maximum is spread over a longer period and is observed 

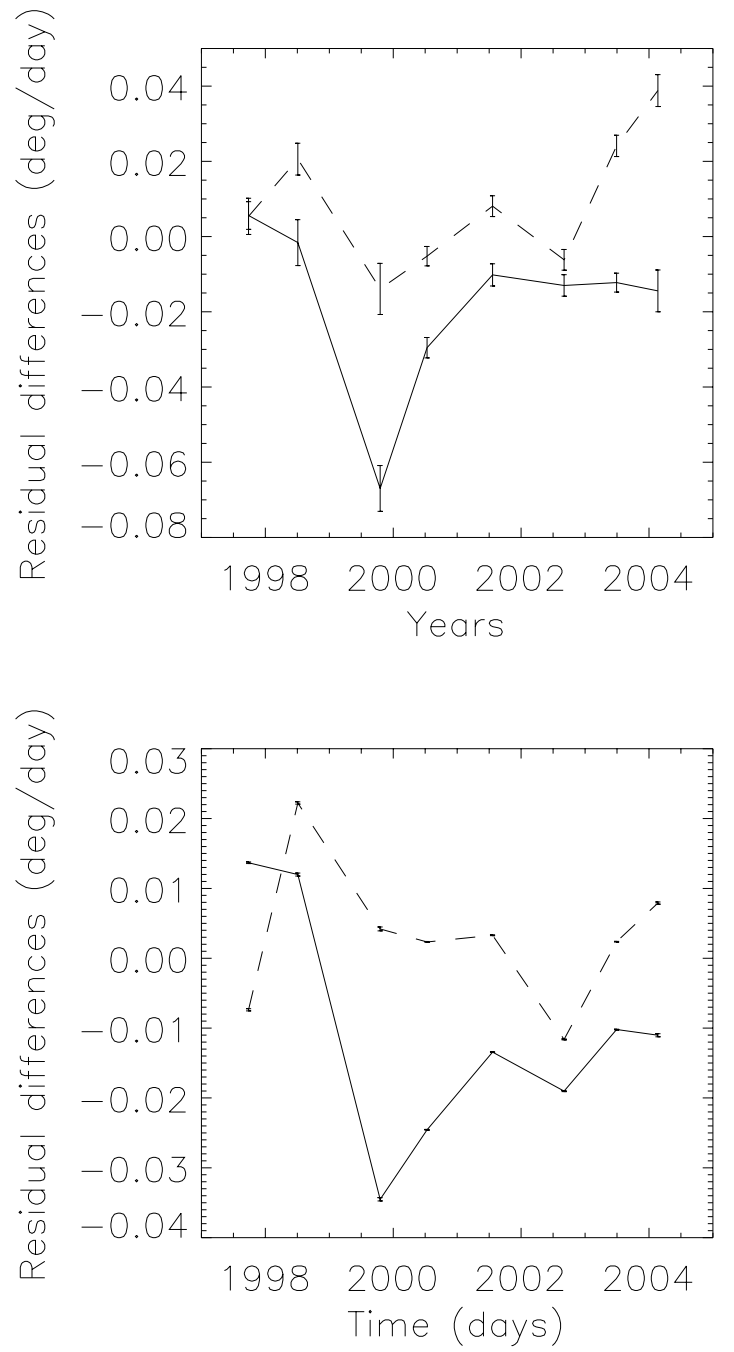

Fig. 10. Difference between the meridional circulation residual for following and leading polarity in the Northern hemisphere (solid line) and Southern hemisphere (dashed line), versus time. Top: averages computed for $\left|\left\langle B_{\mathrm{c}}\right\rangle\right| /\left\langle\left|B_{\mathrm{c}}\right|\right\rangle$ in the range 0.2-0.7. Bottom: position of the Gaussian fit on the distribution of residuals, in the same conditions.

mostly for CT. It also has a larger amplitude at cycle maximum than at cycle maximum (for CT), which is not the case for sunspots. Howard (1990) found plage rotations similar to that of sunspots, with two sharp peaks in the rotation as well. One should note that Meunier et al. (1997a) found a larger rotation of plage and network structures at cycle maximum, while Komm et al. (1993a) found a constant equatorial rotation.

Komm et al. (1993b) found that the meridional circulation maximum amplitude was larger at cycle maximum compared to cycle minimum by about $30 \%$. On the other hand, Meunier (1997b) found that the meridional circulation of plages and network features was larger at cycle maximum compared to cycle minimum (but more or less constant in the Southern hemisphere). They do not compare easily with the results presented in this paper. However both these studies concerned previous cycles, so the comparison may not be valid and it is possible that meridional circulation do not show a systematic behavior from one cycle to the other.
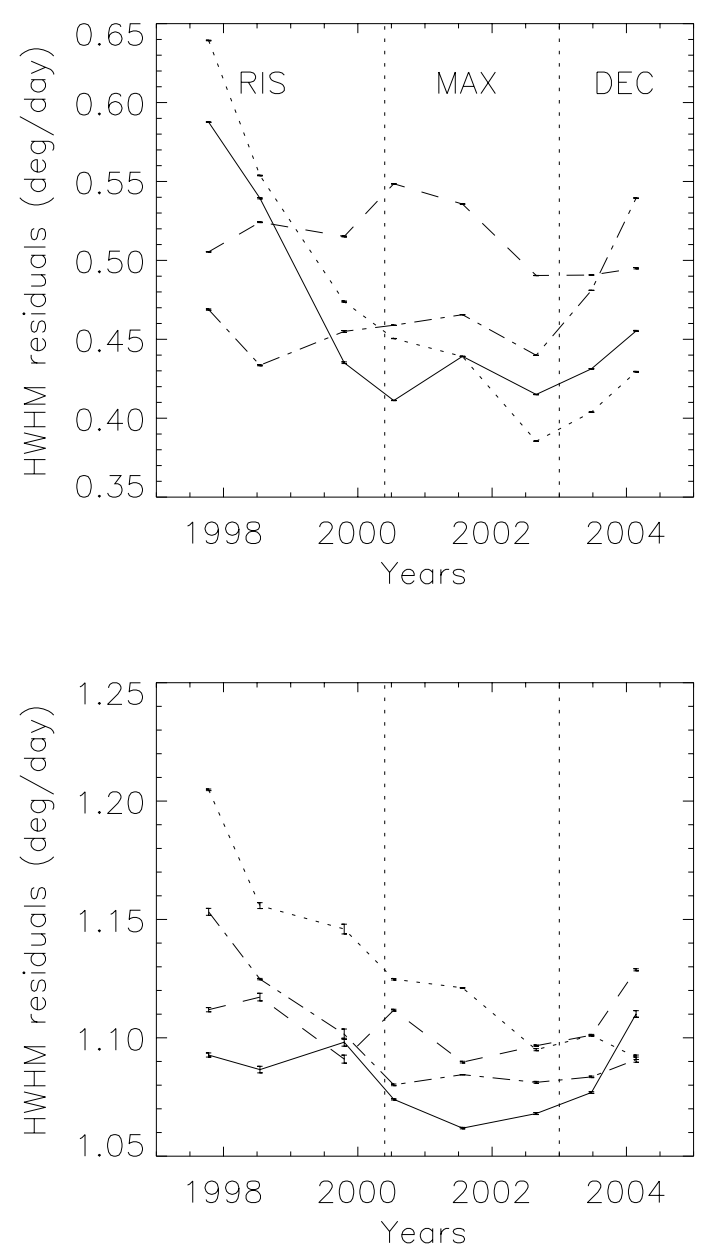

Fig. 11. Top: HWHM on CT meridional circulation residuals distributions versus time, for the leading polarity in the Northern hemisphere (solid line), following polarity in the Northern hemisphere (dashed line), leading polarity in the Southern hemisphere (dotted line) and following polarity in the Southern hemisphere (dotted-dashed line). Bottom: same for the FT method.

On the other hand, the variations in sunspot meridional circulation have been difficult to study. Many works did not find any variation with the phase of the solar cycle or any variation at all (e.g. Balthasar \& Wöhl 1980; Howard \& Gilman 1986). Several authors (Arévalo et al. 1982; Hanslmeier \& Lustig 1983; Lustig \& Hanslmeier 1986) found only variations from one cycle to the other. This was confirmed by Javaraiah (1999) who also found some variation during the solar cycle, with a meridional circulation close to zero during the rising phase of the cycle, and a small equatorward motion during the declining phase. Furthermore, he has detected a anticorrelation of these variations from one cycle to the other. This means that the comparison of various results from different cycles may not be valid. The results obtained here for the magnetic network confirm that the meridional circulation is very complex and may not exhibit a systematic behavior, but the general behavior is different.

The magnetic network dynamics is therefore in agreement with certain results obtained with sunspots previously, such as the more rigid rotation during cycle maximum and two peaks in the equatorial rotation rate. This agreement is mostly 
qualitative, however, since the more rigid rotation and higher equatorial rotation rates during maximum seems to cover a larger period when using the magnetic network. With the magnetic network, it has also been possible to investigate higher latitudes. The new detection of a strong correlation between the Legendre polynomial coefficients should strongly constrain the interpretation of these variations.

The magnetic network analysis also provides new results concerning the variations in the dynamics. Not only has the dynamics versus latitude been studied, but also the dependence on feature size, on magnetic field and on polarity. The distribution in velocity also exhibits some interesting variations with time. A common property of these approaches is that the dynamics are much more variable with time when using crosscorrelation than when using feature tracking, except for the differential rotation. Despite the difficulty in attributing an anchoring depth to each type of approach (Meunier 2005a), it is possible that the dynamics derived from FT may be anchored deeper than CT dynamics: this was derived from the rotation versus depth derived from helioseismology and the larger rotation of FT structures compared to CT. Note that both CT and FT correspond to the same magnetograms formed at the same height (in the range $200-400 \mathrm{~km}$ above the photospheric level, Jones 1989; Meunier \& Kosovichev 2003). This would mean that most of the temporal variations may be present in a very shallow layer close to the surface. It is also puzzling to see that the differential rotation is more variable with FT, while the other characteristics, such as the rotation residuals as a function of the structure size, are very stable. The opposite is observed for CT.

For that reason, it was very interesting to compare our data to recent results using time-distance helioseismology for various depths (Chou \& Dai 2001; Haber et al. 2002; Basu \& Antia 2003; Zhao \& Kosovichev 2004). The North-South asymmetry of the meridional circulation at high latitude significantly decreases over the cycle, and this is in good agreement with the result of Basu \& Antia (2003). However, they observed a decrease in the meridional circulation with time in both hemispheres, as is the case here. Their asymmetry variation comes from the fact that the decrease is stronger in the Northern hemisphere, so the agreement is not complete. Their variation in amplitude of meridional circulation is visible down to $0.985 R_{\odot}(10.5 \mathrm{Mm})$ and the meridional circulation variations are more complex below this value. On the other hand, Zhao \& Kosovichev (2004) have studied the period 1996-2002 using a time-distance analysis for two ranges of depths, 3-4.5 Mm and 6-9 $\mathrm{Mm}$. Close to the surface and at latitude $50^{\circ}$ they found an increase followed by a decrease towards zero, while they found a very small decrease in the Southern hemisphere. Deeper in the convective zone, only decreases are seen. Therefore, their results may match our result in the Northern hemisphere to some extent but not in the Southern hemisphere. At lower latitude, in the range $30-40^{\circ}$, they observed a significant decrease that we do not observe. Haber et al. (2002) have also used a time-distance analysis for data between 1996 and 2001. They have extracted the meridional circulation in two ranges of depths as well, around $0.9 \mathrm{Mm}$ and 7.1 Mm deep. Close to the surface and around latitude $50^{\circ}$, an increase in meridional circulation with time is not compatible with our observation in the Northern hemisphere, while a constant meridional circulation in the Southern hemisphere does not fit with our results either. Deeper, they found a cell reversal in the Northern hemisphere which we do not observe. Chou \& Di (2001) have determined the meridional circulation between 1994 and 2000 for depths in the ranges 9-26 Mm and 26-43 Mm. For the second half of the period they found a tendency for an increase in meridional circulation at high latitude in both hemispheres, so here again this does not fit our results.

This comparison with helioseismology shows that the meridional circulation derived from this technique leads to different temporal variations than the FT and CT measurements. Several limitations may be at the origin of this discrepancy. First, the anchoring depth may be different, and Basu \& Antia (2003) showed how the variation in meridional circulation was variable with depth. Furthermore, the processes at the origin of the difference between the dynamics close to the surface and the CT and FT dynamics (Meunier 2005a) are not known, and they may vary with time. Also, the temporal coverage for a given year may be different. Here most of the year is covered (except for a large gap in 1998), while helioseismological studies have often used shorter time intervals, down to $30 \%$ coverage only.

The variations in leading-following differences (discovered in Meunier 2005b) are quite different from that deduced from the Zhao et al. (2004) analysis using time-distance analysis to derive the flows. Because such a difference was observed with CT but not with FT, Meunier (2005b) concluded that this may be a different phenomenon. The larger amplitude observed by Zhao et al. (2004) suggests that their leading-following differences may contain a contribution related to our CT differences. Note that FT, CT and time-distance analysis correspond to different sensitivities to the layers below the surface. This is relatively well-known for the time-distance but it is not the case for CT and FT (Meunier 2005a). It is therefore difficult to conclude about a common physical process at the origin of the two observations. Our present observation is in good agreement with this conclusion. The following-leading difference in meridional circulation also exhibits some temporal variations, which are relatively well correlated between the hemispheres despite the strong North-South asymmetry. These variations could be related to the polar reversal in the magnetic field.

In general, the meridional circulation tends to exhibit a more complex behavior than the rotation. The strong NorthSouth asymmetry of the meridional circulation variation shows that it is very difficult to derive a simple cyclic behavior (with an 11 year period) for the meridional circulation as opposed to the rotation. However, the width of the distribution in both velocities also exhibits a complex behavior, especially when considering the different polarities with $\mathrm{CT}$, while the variations are simpler when using FT. The CT variations indicate a shift between the two hemispheres of about a year, as the Northern hemisphere is in advance. We know that the polar magnetic field reversal occurred first for the Northern hemisphere and one year later in the Southern hemisphere (Harvey \& Recely 2002; Wang et al. 2002) and at approximately the same epoch than the typical period derived in this study. Therefore, this 
distribution in velocity could be related to the polar magnetic field reversal. What we do observe is that large-scale flows associated with the following polarity regions (which play an important role in the magnetic field reversal) increase faster with time in the hemisphere where the reversal occurs first. It is quite likely that the large-scale circulation associated with the following polarity regions plays a role in this reversal.

Acknowledgements. SOHO is a mission of international cooperation between the European Space Agency (ESA) and NASA.

\section{References}

Ambrož, P. 2001, Sol. Phys., 198, 253

Arévalo, M. J., Gomez, R., Vázquez, M., Balthasar, M., \& Wöhl., H. 1982, A\&A, 111, 266

Balthasar, H., \& Wöhl., H. 1980, A\&A, 92, 111

Balthasar, H., Vazquez, M., \& Wöhl, H. 1986, A\&A, 155, 87

Basu, S., \& Antia, H. M. 2003, ApJ, 585, 553

Chou, D.-Y., \& Dai, D.-C. 2001, ApJ, 559, L175

Gilman, P. A., \& Howard, R. F. 1984, ApJ, 283, 385

Haber, D. A., Hindman, B. W., Toomre, J., et al. 2002, ApJ, 570, 855

Hanslmeier, A., \& Lustig, G. 1986, A\&A, 154, 227

Harvey, K. L., \& Recely, F. 2002, Sol. Phys., 211, 31

Hathaway, D. H., \& Wilson, R. M. 1990, ApJ, 357, 271

Howard, R., \& Gilman, P. A. 1986, ApJ, 307, 389
Javaraiah, J. 1999, Sol. Phys., 189, 289

Jones, H. 1989, Sol. Phys., 120, 211

Kambry, M., \& Nishikawa, J. 1990, Sol. Phys., 126, 89

Komm, R. W., Howard, R. F., \& Harvey, J. W. 1993a, Sol. Phys., 145,

Komm, R. W., Howard, R. F., \& Harvey, J. W. 1993b, Sol. Phys., 147, 203

Lustig, G. 1983, A\&A, 125, 355

Lustig, G., \& Hanslmeier, A. 1987, A\&A, 172, 332

Meunier, N., Nesme-Ribes, E., \& Grosso, N. 1997a, A\&A, 319, 673

Meunier, N., Nesme-Ribes, E., \& Collin, B. 1997b, A\&A, 319, 683

Meunier, N. 1999, ApJ, 527, 967

Meunier, N. 2003, A\&A, 405, 1107

Meunier, N., \& Kosovichev, A. 2003, A\&A, 412, 541

Meunier, N. 2005a, A\&A, 436, 1075

Meunier, N. 2005b, A\&A, 437, 303

Meunier, N. 2005c, A\&A, in press

Nesme-Ribes, E., Ferreira, E. N., \& Mein, P. 1993, A\&A, 274, 563

Nesme-Ribes, E., Meunier, N., \& Vince, I. 1997, A\&A, 321, 323

Scherrer, P., Bogart, R. S., Bush, R. I., et al. 1995, Sol. Phys., 162, 129

Snodgrass, H. B., \& Ulrich, R. K. 1990, ApJ, 351, 309

Vazquez, M., \& Wöhl, H. 1986, A\&A, 155, 87

Wang, H. 1988, Sol. Phys., 116, 1

Wang, Y.-M., Sheeley, N. R.Jr., \& Andrews, M. D. 2002, J. Geophys. Res., 107, 1465

Zhao, J., Kosovichev, A. G., \& Duvall, T. L.Jr. 2004, ApJ, 607, L135

Zhao, J., \& Kosovichev, A. G. 2004, ApJ, 603, 776 